\title{
Identification and co-evolution pattern of stem cell regulator miR394s and their targets among diverse plant species
}

\author{
Ashutosh Kumar, Vibhav Gautam, Pramod Kumar, Shalini Mukherjee, Swati Verma and Ananda K. Sarkar
}

\begin{abstract}
Background: Micro RNAs (miRNAs), a class of small non-coding RNAs, have been implicated in various aspects of plant development. miR394 is required for shoot apical meristem organization, stem cell maintenance and abiotic stress responses in Arabidopsis, where it functions by negatively regulating the transcript level of target LEAF CURLING RESPONSIVENESS (LCR), which is an F-box protein-coding gene. The evolutionary conservation of stem cell regulatory miR394-LCR module among plants remains elusive.
\end{abstract}

Results: Our study has identified 79 miR394 and 43 target sequences across 40 plant species using various homology based search tools and databases, and analysed their co-evolution pattern. We customised an annotation workflow which computationally validates 20 novel miR394s from 14 plant species. Independent phylogenetic trees were reconstructed with precursor MIR394s, mature miR394s, and their target sequences along with complementary miR394 binding sites. The phylogeny revealed that mature sequences of miR394s as well as their targets belonging to the F-box protein encoding gene families, were highly conserved. Though, miR394-3p were complementary to miR394s/miR394-5p, they clustered separately.

Conclusion: The existence and separate clustering of miR394-3p and miR394s/miR394-5p indicate their independent regulation. The phylogeny also suggests that miR394s had evolved at the beginning of gymnospermangiosperm divergence. Despite strong conservation, some level of sequence variation in miR394s and the complementary binding sites of their targets suggests possible functional diversification of miR394-LCR mediated stem cell regulation in plants.

Keywords: miR394, LCR, Small RNA, Phylogenetic analysis, miRNA evolution

\section{Background}

MicroRNAs (miRNAs), a class of small non-coding RNAs, have been reported to regulate various aspects of plant development and physiological processes [1-4]. miRNAs function by negatively regulating their target genes mostly through cleavage at mRNA complementary site or by translational inhibition $[1,4,5]$. Often, a family of similar small (21 to 24 nucleotides) functional mature miRNA(s) may be produced through the processing of a single or multiple precursor MIRNAs (relatively longer) of the same family gene(s) $[1,6,7]$. Novel miRNAs evolve through events like gene duplication and de novo

\footnotetext{
* Correspondence: aksarkar@nipgr.ac.in

National Institute of Plant Genome Research, Aruna Asaf Ali Marg, New Delhi 110067, India
}

emergence from different genomic locations and repress specific genes. Young miRNA families have a high evolutionary rate as compared to the primitive ones [8]. A comprehensive understanding of the origin and functional divergence/convergence of these small RNAs can provide cues about the evolution of their target preference.

Unlike protein coding genes, the mature miRNAs and their target complementary mRNA sequences are critical in deciding the functional diversification. Sequence variation in mature miRNA or its complementary sequences may lead to the functional diversification of miRNA mediated gene regulation and its developmental or physiological outcome [9]. Moreover, the miRNA-target interactions are highly conserved, though some studies have predicted and validated non-conserved targets of miR166 and miR167 [9-11]. miR165/166, which generally negatively regulates 
the transcripts of $H D$-ZIPIII family in a conserved manner, was also shown by us to target non-HD-ZIPIII transcripts in Physcomitrella, a functional change caused by critical sequence variation [9]. Similarly, we have shown earlier that critical sequence variation in mature miR167 and its complementary binding sites at target mRNA created novel target like CALCINEURIN B-LIKE10 (CNBL10) in apple, besides conserved targets $A R F 6 / 8[10,11]$. This kind of change often leads to the evolution of novel targets, henceforth leading to functional diversification of miRNAs mediated regulation [11].

Unlike animals, the post-embryonic development in plants is uniquely regulated, which is marked by continuous activity of shoot and the root meristems leading to the formation of distinct shoot and root systems [12]. The plant shoot apical meristem (SAM) harbours a self-perpetuating population of pluripotent stem cells and their organizing centre, which are maintained by activity of several key genes and phytohormones $[13,14]$. Besides protein coding genes, a few miRNAs, such as miR165/166 and miR394 have been implicated in shoot meristem regulation in Arabidopsis [4]. In Arabidopsis, the protoderm derived miR394, which are processed from two precursors MIR394a/b, moves to the distal meristem during embryonic shoot meristem formation by negatively regulating the transcripts of its target LEAF CURLING RESPONSIVENESS $(L C R)$ which belongs to F-box protein family [6, 15-17]. The miR394-LCR regulatory module is also implicated in abiotic stress responses in Arabidopsis $[18,19]$. Some other F-box protein encoding genes, like TRANSPORT INHIBITOR RESPONSE1 (TIR1) and MORE AXILLARY GROWTH2 (MAX2) are also negatively regulated post-transcriptionally by miR393 and miR528, respectively $[16,20,21]$. Interestingly, miR394 only targets one F-box protein gene $L C R$ (At1 g27340) in Arabidopsis. Since miR394-LCR module play important physiological and developmental role, it is imperative to study its presence and evolutionary pattern among diverse plant species Therefore, a comprehensive analysis of co-evolutionary pattern of miR394 and its targets ( $L C R$ or non- $L C R)$ is required to elucidate the functional conservation and/or divergence of miR394-LCR/target module, an important component of stem cell regulation, across plant kingdom. This knowledge may further help in understanding the role of miR394-target in other plant species of interest.

Here we identified and retrieved MIR394 genes and also predicted, annotated and validated novel miR394s. These were used for the reconstruction of separate phylogenetic trees for precursor and mature sequences along with their respective conserved/non-conserved targets. Our phylogenetic analysis suggests that the mature miR394s were highly conserved than their respective precursor MIR394 sequences. Subsequently, the phylogeny of identified conserved and non-conserved targets suggested some level of functional diversification, despite strong conservation of miR394-target module in plants. The presence of independent miR394-3p sequences targeting non-conserved genes may also contribute to functional diversification.

\section{Results}

Identification of precursor and mature sequences of miR394s miR394 plays a crucial role in the shoot meristem development by regulating the spatial organization of stem cell niches. Therefore, it is imperative to further elucidate the regulatory roles played by miR394s across the plant kingdom. The availability of well annotated complete genome sequences of diverse angiosperms has enabled us to draw genetic relationship between the MIR394 gene family and their targets across the angiosperms.

Though MIR394 is a small and less studied gene family, we retrieved 59 mature miR394 sequences from 26 different plant species available in miRBase Registry database (http:// www.mirbase.org/) (Table 1). Among these 59 sequences, 48 sequences were found to be processed from $5^{\prime}$ end, whereas 11 sequences were processed from 3 ' end of the stem-loop precursors (Table 1). The highest number of mature miR394 sequences i.e., 9, were retrieved form the Glycine max out of which, 7 were processed from the $5^{\prime}$ end and rest two were processed from the $3^{\prime}$ end of the stem-loop precursor. We however, did not get any homologs of MIR394 in other plant species except angiosperms. The already registered and newly annotated miR394 sequences were retrieved from the plant species under study and subjected to sequence similarity and uniqueness analysis by multiple sequence alignment (MSA) using ClustalX2 (Fig. 1). The multiple sequence alignment (MSA) showed that most of the mature miR394 sequences share maximum identity except those which were processed from 3' end of the stem-loop precursors (Fig. 1). The statistical significance of aligned miR394 sequences calculated through Kolmogorov-Smirnov statistical test, suggested that the $\geq 0.3$ fraction of mature miR394 sequences have $\geq 90 \%$ sequence identity (summarized in Additional file 1; Figure S1A). Whereas, $\sim 0.2$ fraction of the precursor pre-MIR394 sequences have $>55 \%$ sequence identity (summarized in Additional file 1; Figure S1B). Our analysis hereby indicates that in spite of showing a good similarity, the mature miR394s are more conserved than their precursor sequences.

\section{The newly annotated MIR394 family members}

In the past few years, the advent of sequencing technologies has led to the availability of whole genome sequences of a number of plant species in public databases. Apart from miR394s sequences registered in miRBase, we also identified some more homologs of MIR394 sequences from the newly sequenced genomes available in the NCBI database. These homologs of MIR394, were subjected to a computational workflow which involved the prediction, in silico validation, 
Table 1 List of miR394s retrieved from miRBase. Species specific sequences of miR394s retrieved from miRBase. The list contained the number of miR394 which were specifically processed either from 5' end (miR394/miR394-5p) or 3' end (miR394-3p) of stem loop precursors

\begin{tabular}{|c|c|c|c|c|}
\hline miRBase IDs & Name of plant species & No. of miR394/miR394-5p & No. of miR394-3p & Total No of miR394s \\
\hline ahy-miR394 & Arachis hypogaea & 1 & 0 & 1 \\
\hline aly-miR394 & Arabidopsis lyrata & 2 & 2 & 4 \\
\hline ata-miR394 & Aegilops tauschii & 1 & 1 & 2 \\
\hline ath-miR394 & Arabidopsis thaliana & 2 & 1 & 3 \\
\hline atr-miR394 & Amborella trichopoda & 1 & 0 & 1 \\
\hline bdi-miR394 & Brachypodium distachyon & 1 & 0 & 1 \\
\hline bna-miR394 & Brassica napus & 2 & 0 & 2 \\
\hline cca-miR394 & Cynara cardunculus & 1 & 0 & 1 \\
\hline cme-miR394 & Cucumis melo & 2 & 0 & 2 \\
\hline cpa-miR394 & Carica papaya & 2 & 0 & 2 \\
\hline csi-miR394 & Citrus sinensis & 1 & 0 & 1 \\
\hline ghr-miR394 & Gossypium hirsutum & 2 & 0 & 2 \\
\hline gma-miR394 & Glycine max & 7 & 2 & 9 \\
\hline lus-miR394 & Linum usitatissimum & 2 & 0 & 2 \\
\hline mdm-miR394 & Malus domestica & 2 & 0 & 2 \\
\hline mes-miR394 & Manihot esculenta & 2 & 0 & 2 \\
\hline nta-miR394 & Nicotiana tabacum & 1 & 0 & 1 \\
\hline osa-miR394 & Oryza sativa & 1 & 0 & 1 \\
\hline ppe-miR394 & Prunus persica & 2 & 0 & 2 \\
\hline ptc-miR394 & Populus trichocarpa & 2 & 2 & 4 \\
\hline sbi-miR394 & Sorghum bicolor & 2 & 0 & 2 \\
\hline sly-miR394 & Solanum lycopersicum & 1 & 1 & 2 \\
\hline ssl-miR394 & Salvia sclarea & 1 & 0 & 1 \\
\hline tcc-miR394 & Theobroma cacao & 2 & 0 & 2 \\
\hline vvi-miR394 & Vitis vinifera & 3 & 0 & 3 \\
\hline zma-miR394 & Zea mays & 2 & 2 & 4 \\
\hline Total & & 48 & 11 & 59 \\
\hline
\end{tabular}

and annotation of miR394 sequence structures for the plant species under study. The flowchart (Fig. 2) for the aforesaid computational workflow has been explained in the materials and methods section. A total of 20 new miR394s stem-loop precursors were validated in silico from 14 different species (Table 2). We identified three new paralog sequences of MIR394 from each Camelina sativa and Raphanus sativus. Similarly, two new paralog sequences of MIR394 were identified from each of Eucalyptus grandis, Cucumis sativus and Populus euphratica (Table 2). These findings about the new members of MIR394 family might be helpful in understanding their functional conservation/divergence.

\section{miR394s/miR394-5p sequences are more conserved than miR394-3p}

The phylogenetic study carried out for revealing the genetic relationship between MIR394 genes and mature
miR394s sequences across the plant kingdom were completed using Neighbour-Joining (NJ) and Maximum Likelihood (ML) methods, respectively, with parameters described later. The ML tree for 79 mature miR394 sequences branched off into two groups (group I and II) with high bootstrap value (Fig. 3). The group I had the smallest cluster consisting only of three sequences, whereas group II contained rest of the miR394 sequences. Further, group II was divided into two clades, clades I and II (Fig. 3). The ML tree showed that the group I, and clade I from group II had all the miR394 sequences processed from the $3^{\prime}$ end (miR394-3p). The clade II of group II had only miR394 sequences which were processed from $5^{\prime}$ end (miR394s/miR394-5p). Interestingly, it was observed that the clusters containing miR394-3p sequences had diverged randomly with varied branch lengths (group I and clade I of group II), 

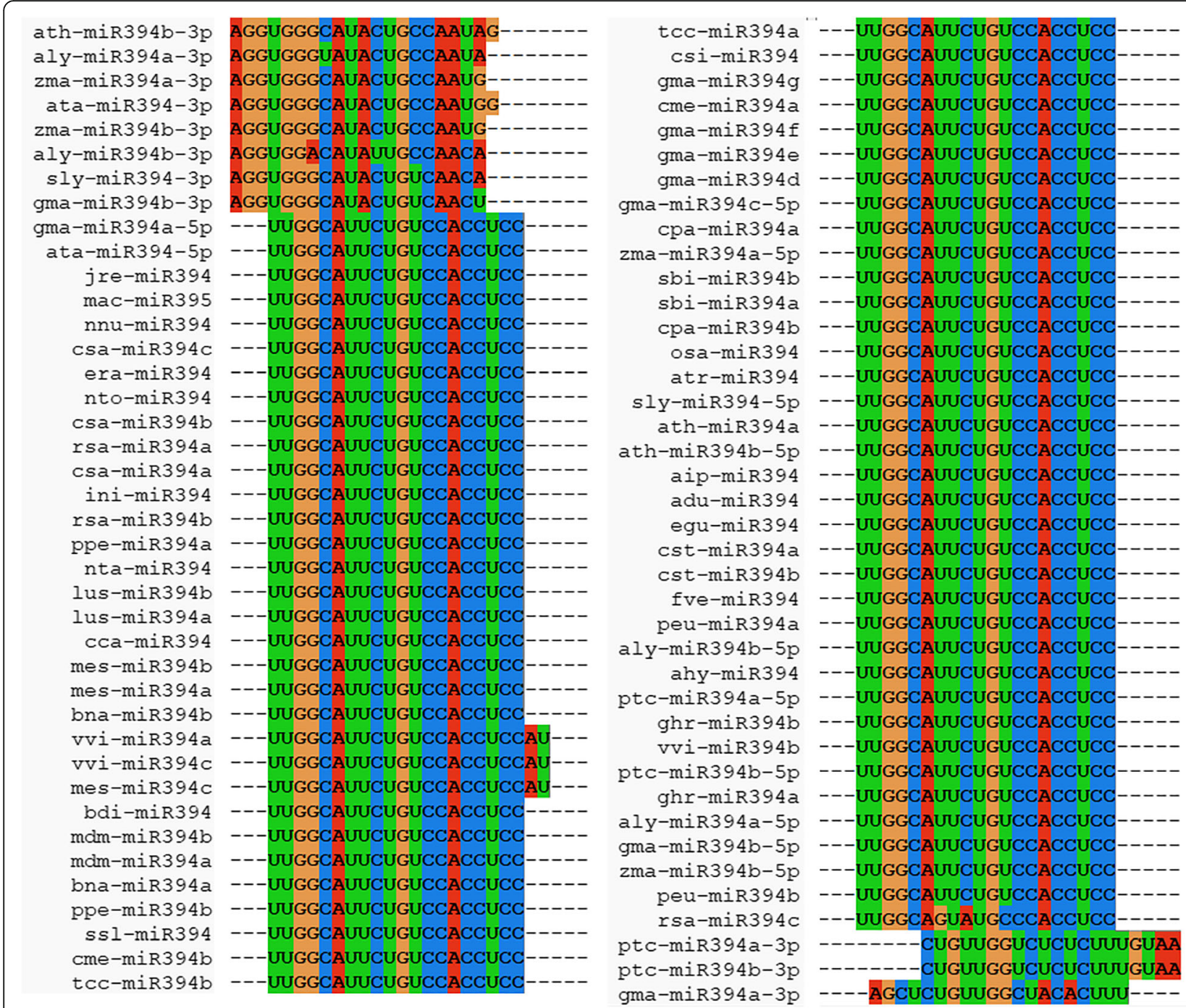

Fig. 1 Multiple sequence alignment of retrieved miR394 sequences from miRBase using ClustalX2. The alignment of retrieved 79 miR394 to identify the uniqueness among the sequences based on homology

however clade II (group II) showed a uniform branch length. Group I in the ML tree had only three sequences ptc-miR394a-3p, ptc-miR394b-3p and gma-miR394a-3p. The ptc-miR394a-3p and ptc-miR394b-3p diverged from the gma-miR394a-3p (Table 1) with a higher rate of substitution, evident from its longer branch length (Fig. 3). Clade I from group II contained the sequences which separated from gma-miR394b-3p. Similar to group I, all the miR394-3p sequences randomly diverged with varied rate of substitution (Fig. 3). The first separation from common ancestor in clade I was shown by sly-miR394$3 p$ followed by aly-miR394b-3p sequence (Table 1). The next separation formed two clusters, one had the sequences zma-miR394a/b-3p and ata-miR394-3p, and the second had aly-miR394a-3p and ath-miR394b-3p (separated with higher rate of substitution) (Table 1). These three sequences, zma-miR394a/b-3p and
ata-miR394-3p, separated with a uniform substitution rate (Fig. 3). Interestingly, clade II in ML tree contained miR394s/miR394-5p sequences, which were uniformly placed with a constant rate of substitution and high bootstrap value, hence signifying a high conservation of these sequences (Fig. 3). The nomenclature of "miR394$3 \mathrm{p}$ ", signifies that these sequences are processed from 3' end of stem loop precursors, complementary to miR394s/miR394-5p. Therefore, a separate ML tree was reconstructed using reverse complementary sequences of miR394-3 ps (miR394-3pRC) along with other miR394s (summarized in Additional file 1; Figure S2). Interestingly, the ML phylogeny revealed that miR394$3 p R C$ along with miR395-3p sequences clustered separately (summarized in Additional file 1; Figure S2) similar to the miR394 ML tree (Fig. 3). Here, all the miR394-3pRC sequences formed separate cluster, except the sequences 


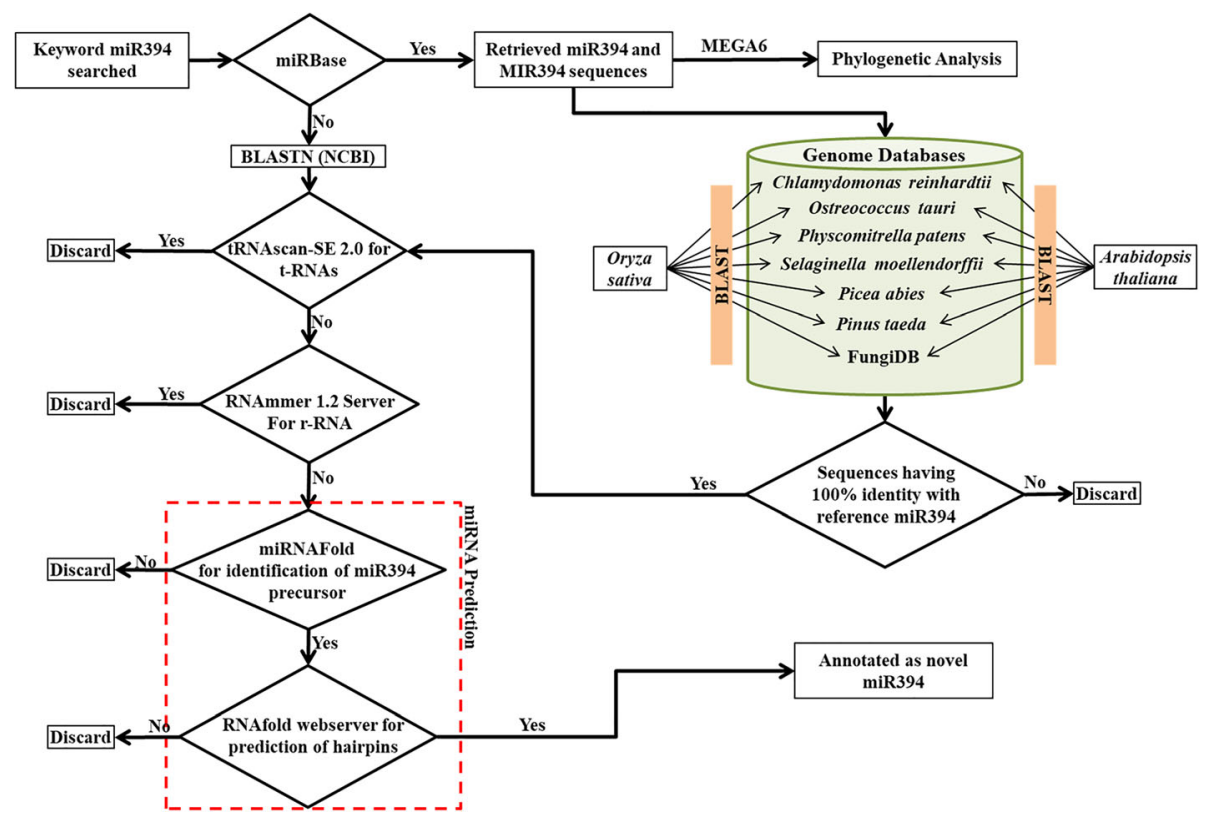

Fig. 2 Flow chart for the prediction of new MIR394 sequences from newly sequenced genome. The known miR394 sequences used to identify and new one using NCBI BLAST. Identified MIR394 sequences further screened for the prediction of possible stem-loop precursor and annotated using different bioinformatics tools

ptc-miR394a/b-3pRC and gma-miR394a-3pRC (summarized in Additional file 1; Fig. S2) which distantly clustered from the miR394-3p sequences with the same topology.

\section{Random divergence was present among precursor MIR394s (pre-MIR394) sequences}

We also analysed the genetic relationship among pre-MIR394s sequences by reconstructing the NJ tree. The NJ tree branched off many times to produce sub-clades with a varied rate of substitution (Fig. 4). All pre-MIR394s were divided into two clusters from a common ancestor (Fig. 4). The first separation in the cluster I was shown by closely related nto-pre-MIR394 and nta-pre-MIR394 (Table 2). Subsequent separation was of rsa-pre-MIR394, which has a longest branch length due to higher substitution rate (Fig. 4, Table 2). In cluster I, rest of the pre-MIR394s sub-divided to form two clades with random distribution, variable branch length and substitution rate. In cluster II, the first divergence was of aly-pre-MIR394 from common ancestor. Further branching placed the clade formed by three pre-MIR394s distantly from other sequences with the highest branch length. This clade contained closely related cca-pre-MIR394 and ssl-pre-MIR394 which separated from era-pre-MIR394 (Tables 1 and 2), have evolved fast evident from higher substitution rate (Fig. 4). Rest of the pre-MIR394s homologs in cluster II were placed randomly and sub-divided into many sub-clades with varied substitution rates. Interestingly, most of monocot pre-MIR394s formed a separate single sub-clade in cluster II. Likewise, most of the newly identified pre-MIR394s also formed a separate single sub-clade with a lower substitution rate and very poor bootstrap value (Fig. 4).

Unlike mature miR394 sequences which were found to be highly conserved, the NJ tree formed by pre-MIR394 sequences showed less conservation which is evident from the formation of multiple branches and clusters, with a very random rate of substitution (Fig. 3). The sequence alignment statistics also supported this randomness among the precursor sequences (summarized in Additional file 1; Figure S1B).

\section{Unique identical miR394s (UmiR394s) majorly target transcripts belonging to F-box gene family}

During the course of evolution and selection, many target genes have undergone sequence variation in the binding site. Therefore, it is imperative to understand the uniqueness of miR394 sequences for targeting transcripts of conserved/diverged gene/s, although having variation at their complementary binding site/s. This study will help in elucidating the co-evolution pattern of miR394, their targets and miR394 mediated gene regulation.

The MSA of the miR394 sequences formed different clusters of mature sequences depending upon their sequence similarity and uniqueness. A total of eight unique clusters were formed (UmiR394-1 to 8). Further, we observed that only one cluster (UmiR394-1) was shared by large number of species with only miR394s/ miR394-5p sequences, one more cluster was found (UmiR394-2) to contain only miR394s/miR394-5p sequences, shared by two species (Table 3). The MSA 
Table 2 List of putative miR394s predicted from newly sequenced genome. The MIR394 sequences from newly sequenced genome were identified through our customized workflow (Fig. 2) and predicted using miRNAFold tool citing their co-ordinates. The putative miR394s of respective MIR394 were validated using RNAfold webserver, which calculates minimum free energy (MFE) of the canonical secondary structure of predicted miRNAs. The nomenclature of the predicted miRNAs was in accordance with miRBase nomenclature system

\begin{tabular}{|c|c|c|c|c|c|c|}
\hline \multirow[t]{2}{*}{ Name of plant species } & \multirow[t]{2}{*}{ Gene Id } & \multirow[t]{2}{*}{ miR394 IDs } & \multicolumn{2}{|c|}{ Predicted Precursor from MIR394 } & \multirow{2}{*}{$\begin{array}{l}\text { Predicted minimum } \\
\text { free energy (kcal/mol) }\end{array}$} & \multirow[t]{2}{*}{ Putative miR394 Sequence } \\
\hline & & & Start Position & End Position & & \\
\hline Arachis duranensis & LOC107481607 & adu-miR394 & 314 & 467 & -63.37 & UUGGCAUUCUGUCCACCUCC \\
\hline Arachis ipaensis & LOC107634751 & aip-miR394 & 340 & 495 & -71.4 & UUGGCAUUCUGUCCACCUCC \\
\hline Camelina sativa & LOC104740735 & csa-miR394a & 509 & 666 & -77.2 & UUGGCAUUCUGUCCACCUCC \\
\hline Camelina sativa & LOC104756391 & csa-miR394b & 408 & 555 & -70.7 & UUGGCAUUCUGUCCACCUCC \\
\hline Camelina sativa & LOC104702369 & csa-miR394c & 544 & 692 & -58.2 & UUGGCAUUCUGUCCACCUCC \\
\hline Cucumis sativus & LOC105435326 & cst-miR394a & 285 & 429 & -51.9 & UUGGCAUUCUGUCCACCUCC \\
\hline Cucumis sativus & LOC105434629 & cst-miR394b & 196 & 350 & -53.9 & UUGGCAUUCUGUCCACCUCC \\
\hline Erythranthe guttatus & LOC105950700 & egu-miR394 & 248 & 407 & -53.3 & UUGGCAUUCUGUCCACCUCC \\
\hline Eucalyptus grandis & LOC104450295 & era-miR394 & 213 & 370 & -52 & UUGGCAUUCUGUCCACCUCC \\
\hline Fragaria vesca & LOC105349875 & fve-miR394 & 538 & 675 & -57.5 & UUGGCAUUCUGUCCACCUCC \\
\hline Ipomoea nil & LOC109181078 & ini-miR394 & 524 & 681 & -67.1 & UUGGCAUUCUGUCCACCUCC \\
\hline Juglans regia & LOC108984513 & jre-miR394 & 507 & 663 & -64.8 & UUGGCAUUCUGUCCACCUCC \\
\hline Musa acuminata & LOC108951711 & mac-miR394 & 155 & 327 & -73.6 & UUGGCAUUCUGUCCACCUCC \\
\hline Nelumbo nucifera & LOC109115150 & nnu-miR394 & 562 & 718 & -46.1 & UUGGCAUUCUGUCCACCUCC \\
\hline Nicotiana tomentosiformis & LOC104110977 & nto-miR394 & 188 & 326 & -49.3 & UUGGCAUUCUGUCCACCUCC \\
\hline Populus euphratica & LOC105109932 & peu-miR394a & 1175 & 1323 & -70.7 & UUGGCAUUCUGUCCACCUCC \\
\hline Raphanus sativus & LOC108816492 & rsa-miR394a & 108 & 261 & -58.5 & UUGGCAUUCUGUCCACCUCC \\
\hline Raphanus sativus & LOC108816348 & rsa-miR394b & 117 & 270 & -57.4 & UUGGCAUUCUGUCCACCUCC \\
\hline Raphanus sativus & LOC108816010 & rsa-miR394c & 1742 & 1842 & -40 & UUGGCAGUAUGCCCACCUCC \\
\hline Populus euphratica & LOC105137284 & peu-miR394b & 1196 & 1344 & -65 & UUGGCAUUCUGUCCACCUCC \\
\hline
\end{tabular}

result showed that rest of the clusters (UmiR394-3 to 8) contained only miR394-3p sequences, whereas, four clusters (UmiR394-3, UmiR394-5 to 7) were shared by two species and two clusters have only one miR394-3p sequence (Table 3). The largest cluster UmiR394-1 has maximum number of miR394 sequences (65) from 40 different species along with maximum number of 11 Unique Target Sites (UTSs) which were predicted to target transcripts from 38 genes. Further, we observed that two clusters UmiR394-2 and 5 have three miR394 sequences from two species each sharing only one UTS with target genes. The UmiR394-3, 6 and 7 have two miR394 sequences, however UmiR394-6 and 7 each have two miR394 sequences from two species, without any UTS. UmiR394-3 has two miR394 sequences from same species with one UTS targeting one transcript (Table 3).

The MSA showed clustering of miR394 sequences into eight UmiR394 clusters according to their sequence similarity, which were further used for the identification of transcripts having complementary binding sites of UmiR394s using psRNAtarget tool. Many a times, psRNAtarget tool did not identify any target for UmiR394s, hence we also used the NCBI BLAST. Altogether, a total of 43 transcripts were identified for UTSs targeted by 8 UmiR394s. Surprisingly, psRNAtarget or NCBI BLAST was unable to identify any UTSs for UmiR394-6 and 7 in plant species. We observed that most of the identified target transcripts were homologs of F-box protein encoding gene family members and rest were either hypothetical or uncharacterized protein coding homologs. Furthermore, psRNAtarget tool predicted the LCR in Arabidopsis (UTS 1a), a validated target of ath-miR394, along three more different targets (UTS $1 \mathrm{~b}$ to $1 \mathrm{~d}$ ). The UTS 1a cluster was shown to target maximum (20) numbers of target genes (Table 3). In crops, three targets were predicted in maize (UTS 1e and 5) and Glycine max (UTS 1a), two target genes in rice (UTS 1e and $1 \mathrm{~g}$ ) and Sorghum bicolor (UTS $1 \mathrm{e}$ and $1 \mathrm{~h}$ ). The three targets identified in maize were transcripts of genes encoding uncharacterized/unannotated proteins, though two of them have domains of F-box protein (predicted using IntroProScan v5; https://www.ebi.ac.uk/ interpro/search/ sequence-search). Likewise, one of the target from rice and Sorghum bicolor (predicted using IntroProScan v5) belonged to F-box protein encoding gene, whereas all 


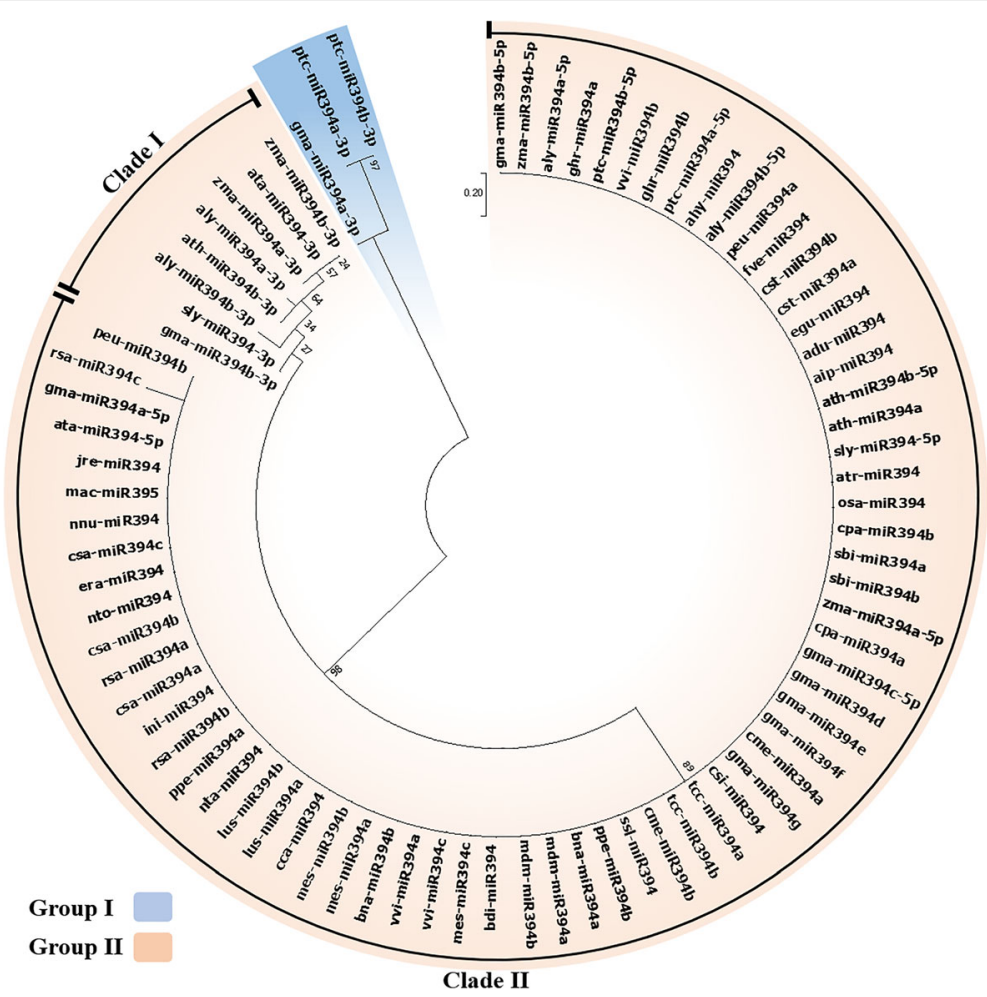

Fig. 3 An unrooted ML phylogeny of miR394s using MEGA6. The tree is divided into two groups group I (represented in blue colour) and group II (represented in pink colour). The group I contains miR394-3p (processed from 3' end of precursor) and group II have miR394/miR394-5p sequences (processed from 5' end of precursor) sequences, also sub-divided into clade I and II. The scale bar represents the nucleotide substitution rate

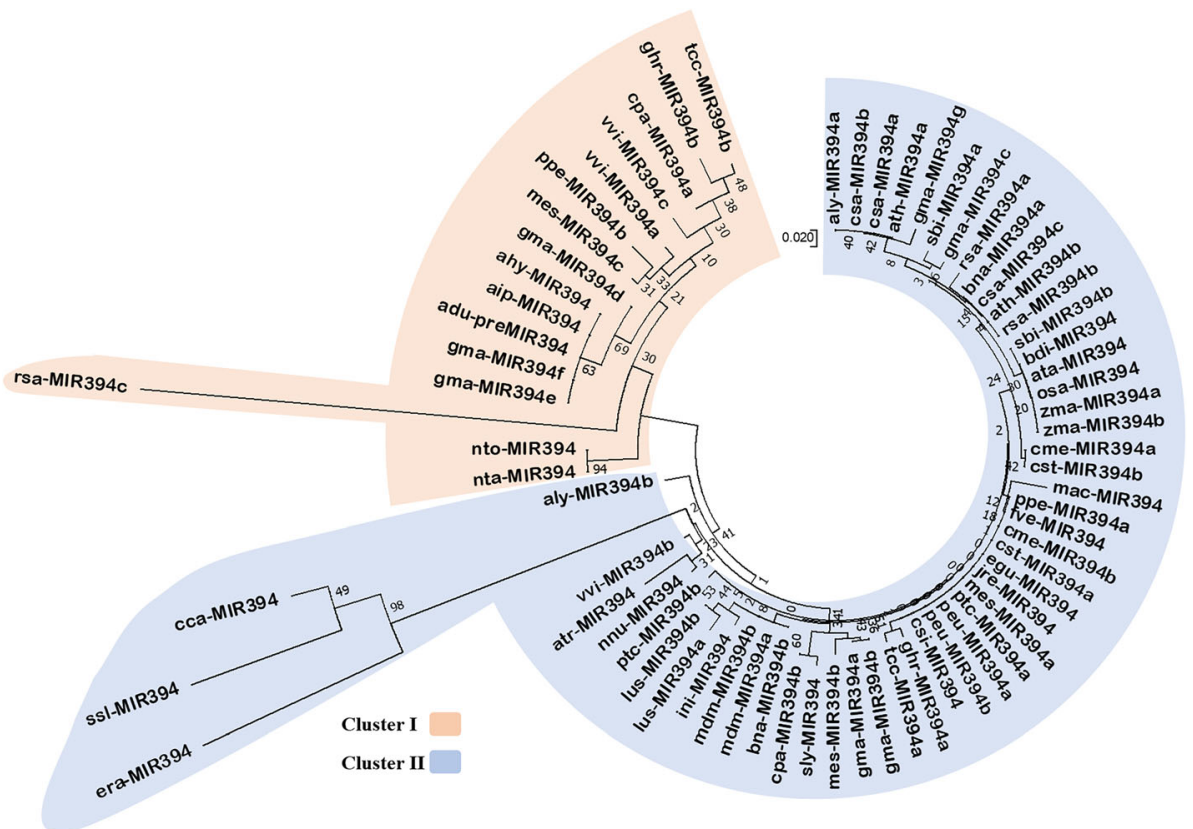

Fig. 4 An unrooted NJ phylogeny of pre-MIR394s using MEGA6. The tree is sub-divided into cluster I and II represented in pink and blue colour, respectively. The pre-MIR394 sequences has shown discrete evolution pattern with varying substitution rate. The scale bar represents the nucleotide substitution rate 


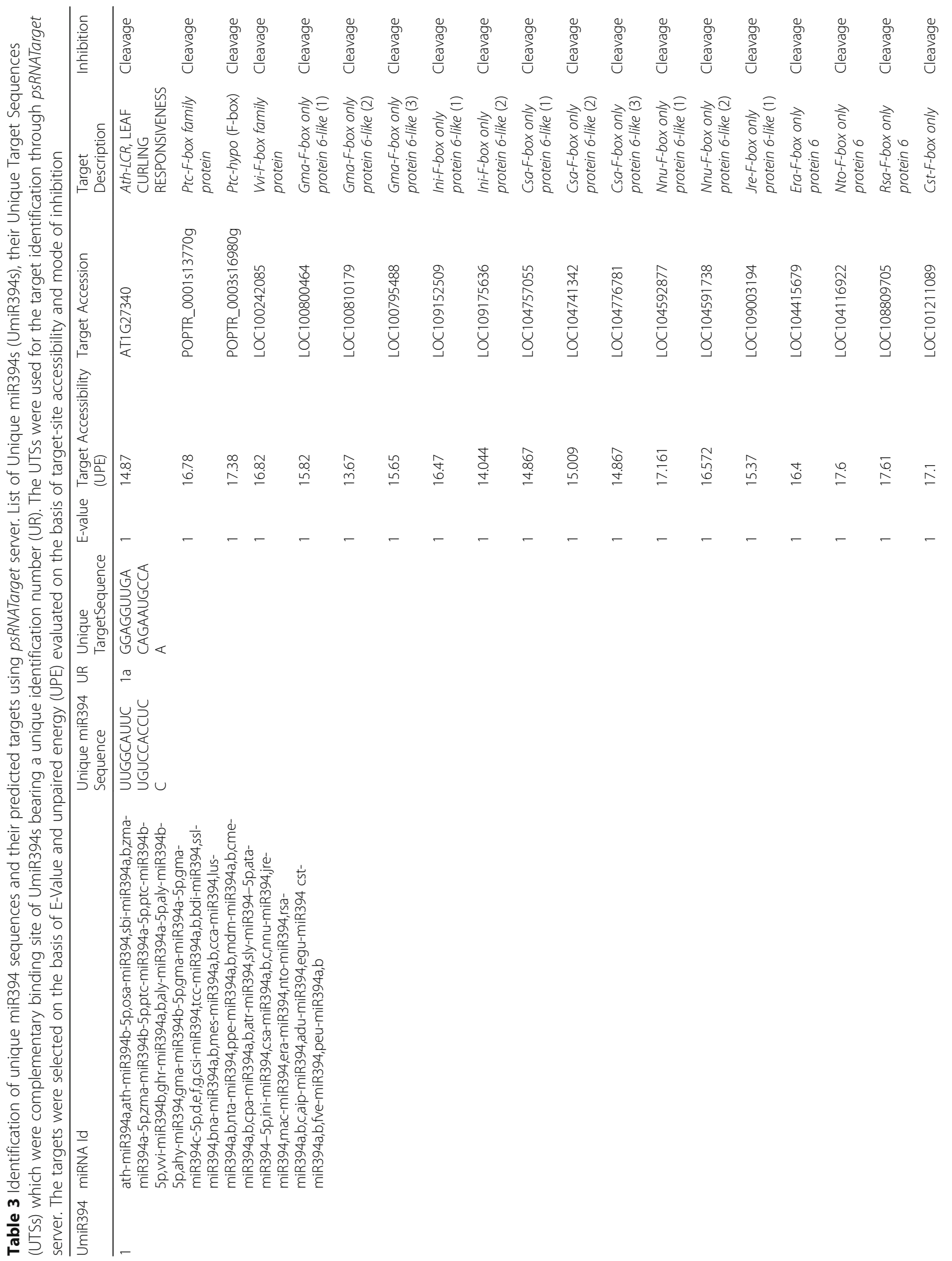




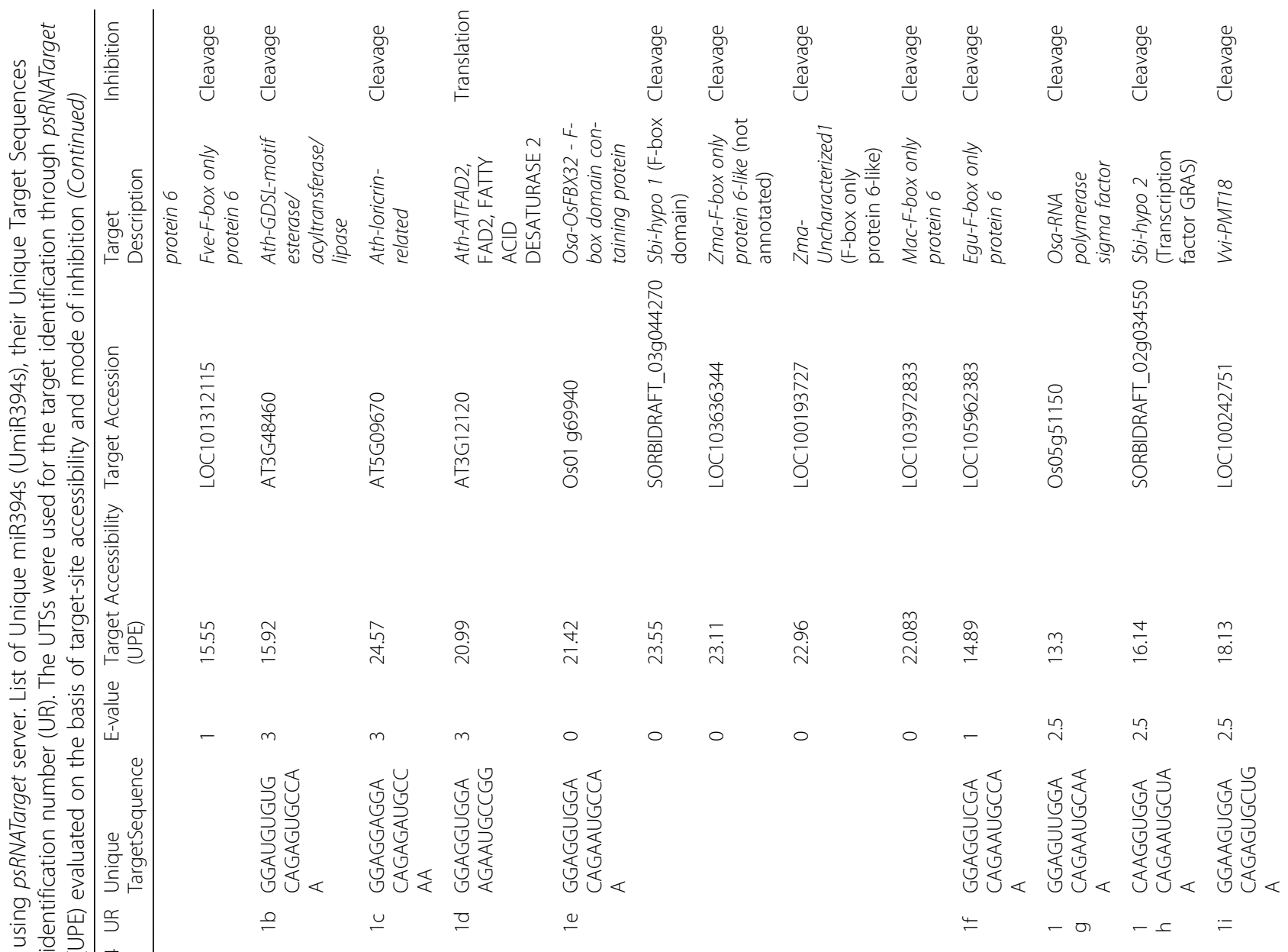


Kumar et al. BMC Evolutionary Biology

(2019) 19:55

Page 10 of 16

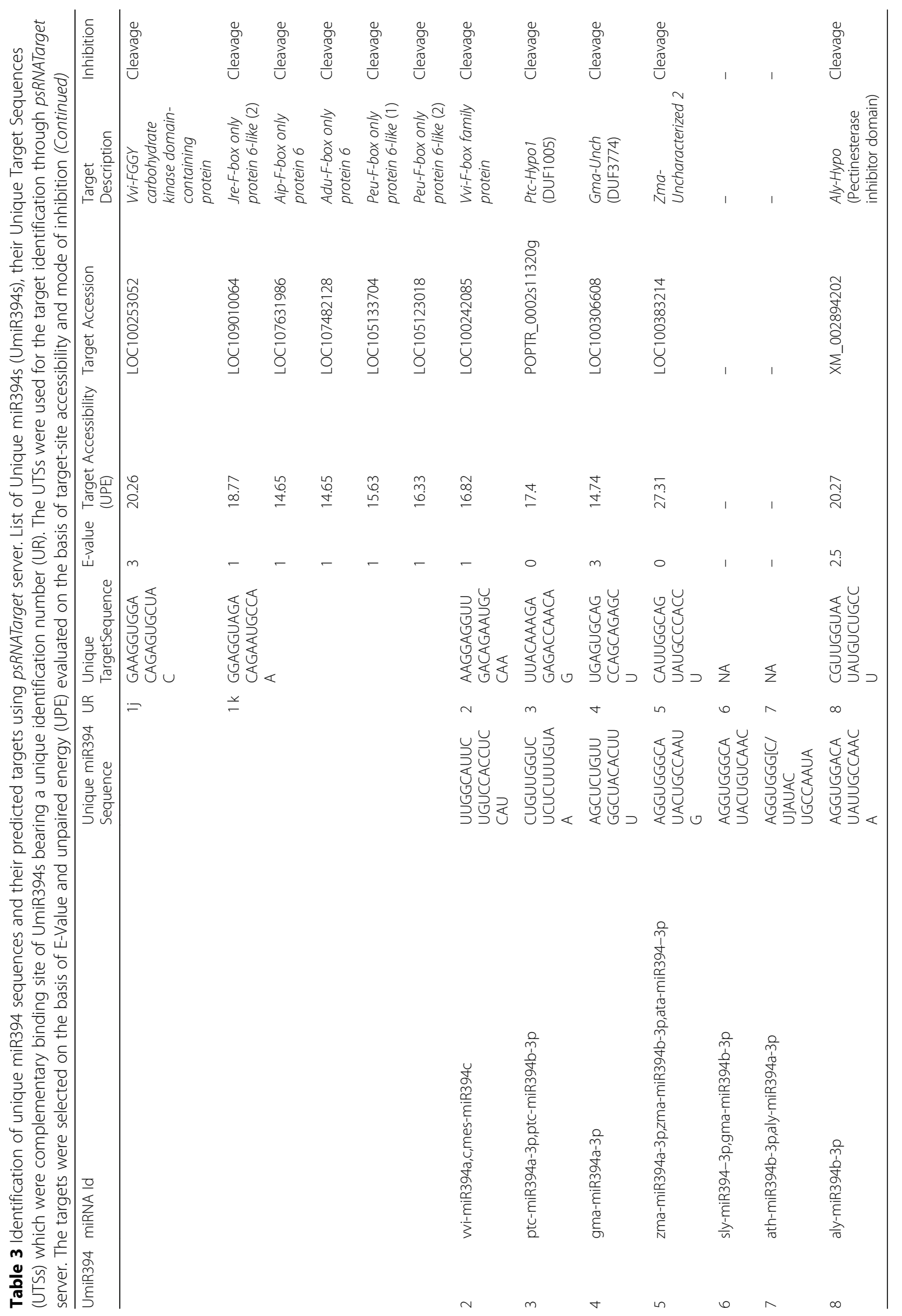


three predicted targets in Glycine max were F-box protein encoding genes (having three different locus) (Table 3).

\section{Phylogenetic analysis revealed strong conservation of targets of miR394s}

The miRNAs and their targets are often found to be conserved, although there are reports about divergence of targets. Therefore, to understand the functional conservation/diversification of miR394s, we studied the genetic relationship by reconstructing a phylogenetic tree from the UTSs and their target gene sequences (Fig. 5). The resultant ML tree was divided into two groups; group I and II. Group I further sub-divided into two clades, and the group II was sub-divided into many clusters (Fig. 5). Though clade I has UTSs which were complementary binding sites of the miR394s/miR394-5ps, it mostly contained non-conserved target genes with a higher rate of substitution, whereas clade II has UTS 5 and 8 closely related with zma-Unch 2 and aly-Hypo (UTSs which were complementary binding sites of the miR394-3p), respectively (Fig. 5, Table 3).
The first divergence from the ancestor in the group II was of UTS $1 \mathrm{~d}$ and its closely related non-conserved ath-ATFAD2 target gene, along with UTS $1 \mathrm{~b}$ closely related with ath-GDSL which is also a non-conserved target gene of miR394 (Fig. 5, Table 3). Subsequent divergence was shown by largest cluster in group II, where the first separation was of UTS 3 and 4 (complementary binding sites of the miR394-3p), closely related with the non-conserved target genes ptc-Hypo and gma-Unch (Table 1), respectively with higher rate of substitution. The rest of the sequences in the group II contained conserved target genes, i.e., the F-box homologs, along with their respective UTSs (complementary binding sites of the miR394s/miR394-5p) which branched off into many sub-clades with lower substitution rate (Fig. 5, Table 3). Some of the conserved targets in the group II were annotated, however most of the target genes were predicted (Fig. 5).

\section{Discussion}

Ubiquitination is a post-translational regulatory process, which controls the activity of numerous proteins during

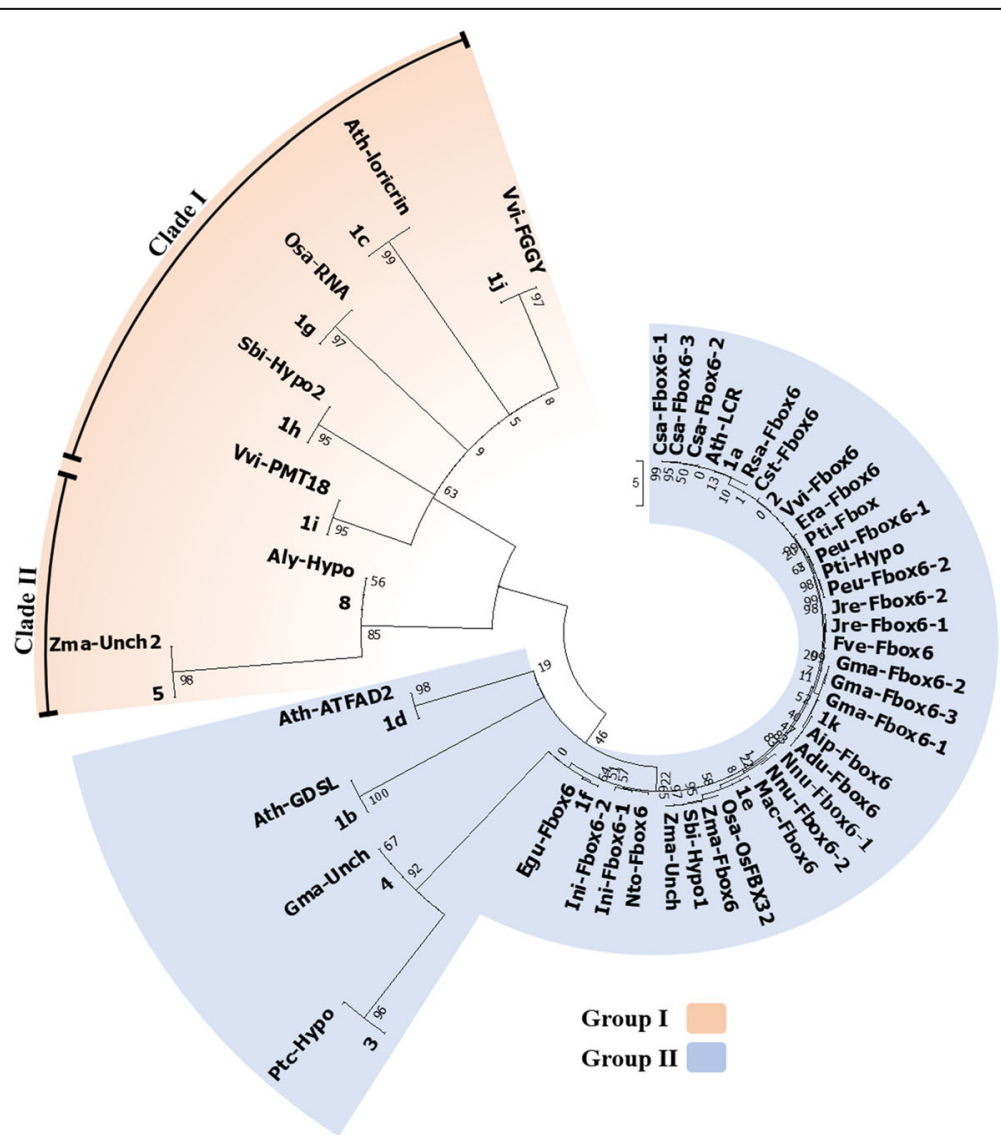

Fig. 5 ML Phylogeny of UTSs and their corresponding predicted target genes using MEGA6. The phylogenetic tree is used for the identification of genetic relationship between UTSs of unique miR394 sequences and their targets. The tree is divided into two groups i.e., group I (represented in pink colour) and II (represented in blue colour), further group I is sub-divided into two clades i.e., clade I and II. The target F-box homologs are highly conserved. The scale bar represents the nucleotide substitution rate 
plant growth and development [22]. The F-box proteins are central component of Suppressor of Kinetochore Protein 1(SKP1)-Cullin (CUL)-F-Box complex [23], where the F-box is attached to SKP1 through its highly conserved amino (N)-terminal ' $\mathrm{F}$-box' motif, leaving the variable C-terminal to bind with different ligands [24]. About 1600 genes in Arabidopsis encode proteins involved in ubiquitination process of which 700 encode F-box subunit $[25,26]$. The $L C R$, belongs to one of the members of the F-box gene family and largely dictates the involvement of the miR394s in various plant processes [17]. Typically, the miR394s are involved in the regulation of SAM organization, fruit and seed development [27], and regulating the abiotic stress responses in Arabidopsis through impairing of pathways involving $L C R$ by post-transcriptional modification $[15,16,19]$. In order to understand the diverse function of miR394 regulating $L C R$, we studied their co-evolution using phylogenetic analysis. While retrieving the known precursor and mature sequences of miR394, we observed that miR394 family comprised very less members/isoforms (Table 1) [28]. The search for new MIR394 homologs from newly sequenced genomes and from other phylum of plant kingdom using our annotation workflow (Fig. 2) [29, 30], revealed a large number of genomes belonging to the different phyla of the plant kingdom which are not yet sequenced. This has created a gap in understanding the evolution of miR394s amongst various plant species. In our study, the retrieved sequences of precursor and mature miR394 belonged to angiosperms, which were used for the reconstruction of phylogenetic tree to identify their genetic relationship and target conservation. Subsequently, the unrooted ML tree of mature miR394s showed less divergence among the miR394 family members across the plant species (Fig. 3). The tree showed two distinct clusters for miR394-3p and miR394/miR394-5p sequences. The cluster of miR394/miR394-5p sequences were found to be highly conserved which was evident from higher homology level and very less substitution rate (due to uniform small branch length in ML tree). This was also evident from the statistical test, Kolmogorov-Smirnov test, which shows $\geq 0.3$ fraction of mature miR394 sequences have $\geq 90 \%$ sequence identity (Additional file $1 \mathrm{~A}$ ). Though miR394-3p sequences formed a separate cluster, they showed a very discrete distribution with higher rate of substitution. This diversification among the miR394-3p sequences might be due to differences in their origin, as they are processed from the $3^{\prime}$ end of stem loop precursors. The miR394-3p sequences are processed from the 3' end of stem loop precursors but are not the exact complements of miR394/miR394-5p, therefore miR394-3p sequences might have shown variation in sequences. A slight change in the sequence of these $20 \mathrm{bp}$ long miRNAs, may significantly contribute in their divergence. This is also supported by our previous studies on miR167 [10]. The ML tree with reverse complementary sequences of miR394-3p (miR394$3 p R C)$ revealed results similar to the previous phylogeny (Additional file 1; Figure S2; Fig. 3). All the miR394/ miR394-5p sequences separated from the common ancestor with a constant rate of substitution (due to higher homology among sequences), whereas the miR394-3p and miR394-3pRC sequences separated with a higher rate of substitution into two clusters. The sequence variation is also evident from the positioning of ptc-mir394a/b-3pRC and gma-miR394-3pRC in the phylogeny (summarized in Additional file 1; Figure S2). These sequences are placed very distantly from their respective miR394-3p sequences, suggesting significant variation between the miR394-3p and miR394/miR394$5 p$ sequences. Additionally, the miR394-3p sequences are processed from the $3^{\prime}$ end (anti-sense) of their precursor sequences, represented as miR394-3p, rather than miR394\%. Therefore, we postulated that not all miR394*s are degraded during the processing of precursor, somewhat they function independently as miR394$3 p$ as indicated in the miRBase (http://www.mirbase. org/), similar to our previous study [10]. Conventionally, the stem loop structure of pri-miRNAs is cleaved by DICER-like proteins to produce mature strands either from $-5 p$ or $-3 p$, where the mature strand (mature miRNA) is responsible for its ability to enter the RISC complex to bind with target transcripts depicting its functionality, whereas the less stable strand is degraded [31]. However, many reports stated about the prevalence of independent $-5 \mathrm{p}$ and $-3 \mathrm{p}$ miRNAs in animal system [32-34], though miRBase reports the presence of both $-3 p$ and $-5 p$ miRNAs in some families. Further, several conserved miRNA-3ps are identified and reported in animal system [34] the regulatory activities of whom are selected and stabilized by natural selection [8].

The number of miR394 sequences in miRBase repository are from very few species and also very less in number, out of these only one species Glycine max has the maximum number of miR394 isoforms (7 miR394s/miR394-5ps and 2 miR394-3ps) (Table 1). All gma-miR394/-5ps are highly conserved and placed in a single cluster (Fig. 3). Further, the gma-pre-MIR394 sequences did not group in a single cluster. They showed a discrete pattern of evolution with a varied rate of substitution, scattered across NJ tree (Fig. 4). This pattern of evolution suggests that gma-pre-MIR394 sequences arose from multiple duplication events, indicating that there has been a prevalence of higher rate of duplication in the Glycine max genome [35].

Exceptionally, we found one sequence atr-pre-MIR394, from species Amborella trichopoda was placed with another dicot pre-MIR394s in cluster II (Fig. 4). The studies 
related to evolutionary relationship of Amborella trichopoda with other angiosperms have categorized Amborella trichopoda as the sole surviving sister species of all other angiosperms [36, 37]. The emergence and evolution of MIR394 gene is observed in the basal angiosperms, at the very beginning of gymnosperm-angiosperm divergence prompting us to suggest that MIR394 genes have evolved exclusively in angiosperms.

The phylogenetic analysis of mature and precursor sequences has shown that the mature ones are more conserved than their precursors. The pre-MIR394s are of longer length than the mature miR394s, making them more vulnerable to mutation/s or other nucleotide changes during evolution causing significant sequence variation. Therefore, we observed random distribution of pre-MIR394 sequences with varied rate of distribution, showing extremely less conservation amongst them. This is evident from the distribution pattern of the highly conserved cca-miR394, ssl-miR394, era-miR394 and rsa-miR394 in the ML tree (Fig. 3, Tables 1 and 2), while their pre-MIR394s are separated distantly with the longest branch lengths, signifying their faster evolution with the highest rate of substitution (Fig. 4).

The targets of miR394s are often conserved divulging its functionality in significant physiological and developmental processes. Thus, to decipher the functional conservation/diversification of miR394, we predicted the targets and reconstructed a phylogenetic tree (Fig. 5). Prior to the phylogenetic analysis, we clustered the mature miR394 sequences using MSA to identify the UmiR394 sequences (Fig. 1). Further, these UmiR394 sequences were used for the identification of their targets and complementary binding sites known as UTSs (Table 3). Sixteen UTSs for eight UmiR394s were identified, targeting forty-three different genes. Most of these target genes belonged to the F-box protein encoding genes (Table 3). Subsequently, the identified UTSs and predicted targets were used for the reconstruction of an unrooted ML tree [10]. The resultant ML tree was divided into many clades (Fig. 5). The UmiR394-1 and -2 contained all the miR394/ mi394-5p members and rest of UmiR394s have miR3943 ps (Table 3). We observed that the UTSs 1a, 1e, 1f, $1 \mathrm{k}$ and 2 are closely related with all the targets which were either F-box or unannotated F-box genes (Fig. 5). Other 12 UTSs were found to be closely related to target gene not belonging to $F$-box, and forming a separate individual clade (Fig. 5). Out of eleven UTSs, four UTSs (UTS 3, 4, 5 and 8) have UmiR394s which consisted of miR394-3ps, which have non-conserved target (Table 3). As evident from the MSA, the miR394-3ps have variation in their sequences (Fig. 1), which resulted into a separate cluster of miR3943ps in phylogenetic tree (Fig. 3). Hence the functional deviation of miR394-3ps was attributed to its $3^{\prime}$ end (anti-sense) processing from precursor sequences causing significant sequence variation. Though non-conserved targets were also identified, our analysis suggest that F-box genes are highly conserved targets of miR394s. The functional diversification of miRNAs by acquiring new targets are due to nucleotide variation in the mature miRNA sequence. The genes may also gain/alter miRNA target sites either by mutations or via segmental duplication, speciation or climatic adaptation [11,38-40]. Some of the miRNAs such as miR167 are reported to cleave non-conserved targets IAA-ALANINE RESISTANT 3 (IAR3) in Arabidopsis [38] and CNBL10 in apple [10]. Similarly, miR827 is also known to targets two different genes, NLA and PHT5 in Arabidopsis and rice, respectively [39]. Extensive analysis of the unannotated paralog F-box genes across the plant kingdom is further required for a better understanding of its evolutionary significance as well as target preference.

\section{Conclusions}

We have identified 79 miR394s, including 20 novel miRNAs, and their target $L C R$ homologs from selected and newly sequenced plant species. The phylogenetic analysis highlights the co-evolutionary pattern of miR394s and their conserved/diverged targets. We observed that the miR394s/miR394-5ps are highly conserved among plants species irrespective of their less conserved MIR394s. Though, miR394-3ps were complementary to miR394s/ miR394-5ps, they clustered separately in the phylogeny suggesting their independent occurrence and a possible justification for their functional diversification. The phylogenetic analysis also suggested that the miR394s had evolved at the beginning of gymnosperm-angiosperm divergence. Further, we identified and computationally validated 20 novel miR394s from 14 newly sequenced genomes using our annotation workflow. LCRs, the member of F-box protein coding genes and conserved target of miR394, were also found to be conserved across different plant species. The phylogeny of miR394 targets and their complementary binding sites have suggested their conservation. Some functional diversification was observed since few of the miR394s were targeting non-conserved targets. Future studies are needed to understand the functional relevance of the selection of non-conserved targets of miR394 in different species.

\section{Methods}

Identification of miR394s and their precursor sequences

To identify the number of miR394 sequences available, we used the miRNA registry database (miRBase version 21, http://www.mirbase.org/). The key word "miR394" was used as query against miRBase to search the miR394 family members in each plant species. Fifty-nine mature miR394 and their precursor MIR394 sequences from various plant species were retrieved. We did not find miR394 from any species other than 
angiosperms (Table 1). The nomenclature for species used for this study is in accordance with miRBase such as for Arabidopsis thaliana as "ath" (Table 1). The miR394 entries in miRBase were further verified using BLAST search in NCBI, (http://www.ncbi.nlm.nih.gov/), and other speciesspecific databases. For phylogenetic analysis, only miRBase registered miR394 were considered and used.

While searching NCBI database, we found some ncRNA sequences having $100 \%$ identity with reference miR394 from newly sequenced genomes. These ncRNA sequences were further scanned using the tools tRNAscan-SE 2.0 (http://lowelab.ucsc.edu/tRNAscan-SE/) and RNAmmer 1.2 server (http://www.cbs.dtu.dk/services/RNAmmer/) for ruling out as t-RNA and r-RNA, respectively [41, 42]. Rest of the ncRNA gene sequences were subjected to the identification of canonical precursor stem-loop structures and in silico validation through publicly available online tools miRNAFold (https://evryrna.ibisc.univ-evry.fr/evryrna/mirnafold) and [43, 44] RNAfold web server (http://rna.tbi.univie.ac.at/cgi-bin/ RNAWebSuite/RNAfold.cgi), a core program of the Vienna RNA package [45]. For the identification of precursor sequences from ncRNA genes miRNAFold was used. The parameters were set as default with the precursor length set at $\leq 170 \mathrm{nt}$. The potential precursors containing miR394 sequences were used for the prediction of secondary structures (stem-loop structure) through RNAfold web server. The RNAfold webserver calculates minimum free energy (MFE) of predicted secondary structure of RNAs using the dynamic programming algorithm [46] and equilibrium base-pairing probabilities are calculated through John McCaskill's partition function (PF) algorithm [47]. The secondary structures were chosen from those having $\geq 18 \mathrm{bp}$ of mature miR394 matching in the folded region followed by a central loop (20 novel miR394s from 14 newly sequenced genomes). Further, each predicted precursor MIR394 sequences from the newly sequenced genome was manually curated and named according to species as used in miRBase database (Table 2).

\section{Phylogenetic analysis and target prediction from unique identical miR394 sequences}

The retrieved miR394 sequences from all the species were aligned together using ClustalX2, and on the basis of this alignment unique identical sequences were identified (Fig. 1, Table 2). Further these unique identical sequences of miR394 were used for target prediction. The statistical significance of sequence similarity/identity of aligned miR394 as well as precursor sequences were calculated through Kolmogorov-Smirnov statistical test in GeneDoc v2.7.0 (summarized in Additional file 1; Figure S1) [48]. Further aligned miR394 sequences were used for studying their comparative evolutionary process by generating phylogenetic tree through MEGA6 [49]. The parameters used for the maximum likelihood (ML) method to generate unrooted tree by considering all the sites in the mature sequences were same as described previously
$[9,10]$. The ML tree was reconstructed by taking gamma distribution with Invariant sites $(G+I)$ for rate calculation having discrete Gamma distribution among sites (4 categories) and GTR Substitution model. The bootstrap calculated for 1000 replicates. The ML tree was inferred through BioNJ as the initial tree using heuristic method $[9,10]$. Additionally, pre-MIR394s were used for the phylogenetic analysis by reconstructing the NJ tree using MEGA6 [10]. The unrooted NJ tree was reconstructed by taking partial deletion with uniform rates and using Maximum Composite Likelihood model. The bootstrap was also calculated for 1000 replicates. The targets of uniquely clustered miR394 sequences (Table 2) were predicted either by psRNATarget tool or BLAST from NCBI. Consecutively, these target genes were also used for phylogenetic analysis with MEGA6. Since the target sequences did not show any consensus during multiple sequence alignment, distance estimation (distance matrix) method was used. Further, the substitution model, p-distance with uniform rates, and pairwise deletion for the gap treatment were used for the reconstruction of phylogenetic tree.

The unique identical miR394 (UmiR394) sequences were used for the prediction of targets using psRNATarget tool [50]. The analysis parameters, maximum E-value and UPE score (maximum energy to unpair the target site) were chosen as 5 and 50 , respectively. The complementary binding sequences UTSs of UmiR394 were identified and numbered (Table 2). These identified UTS were selected on the basis of lowest E-value and UPE score and numbered (as 1, 2, 3 etc.; Table 3). Though, UTS and their corresponding target genes were predicted by psRNATarget tool, some of UmiR394s did not contain any predicted UTS. Therefore, putative target genes were searched for these UmiR394s using NCBI BLAST (http://blast.ncbi.nlm.nih.gov/Blast.cgi) keeping $100 \%$ query coverage and identity, in all available plant genomes. The nomenclature for the target genes used in this study was specified here as "Ath-LCR" to indicate $A$. thaliana $L C R$. Same format was used for target genes from other species (Table 3). Further, these miR394 targets and UTS were used for phylogenetic analysis to understand the evolutionary pattern.

\section{Additional file}

Additional file 1: Figure S1. Statistical analysis using Kolmogorov-Smirnov test through GeneDoc v2.7.0. Calculation of percent identity of aligned miR394 sequences for statistical significance. A. The test has shown that the $\geq 0.3$ fraction of mature miR394 sequences have $\geq 90 \%$ sequence identity B. $\sim 0.2$ fraction of the precursor pre-MIR394 sequences have $>55 \%$ sequence identity. Figure S2. An unrooted ML phylogeny of miR394s along with reverse complementary sequences of miR394-3p using MEGA6. This tree contains reverse complementary sequences of miR394-3p (highlighted, encircled portion). All miRNA394/miR394 - 5 ps are highly conserved with very less substitution rate. The scale bar represents the nucleotide substitution rate. (ZIP 377 kb) 


\section{Abbreviations}

Hypo: Hypothetical; miR394-3pRC: Reverse complementary sequences of miR394-3p; miR394s/miR394-5p: miR394 sequences which were processed from 5' end of stem loop; UmiR394: Unique miR394; Unch: Uncharacterized; UTS: Unique Target Sequence

\section{Acknowledgements}

AK, SM, and SV thank the Department of Science and Technology - Science and Engineering Research Board (DST-SERB) for National-Post Doctoral Fellowship (N-PDF). VG and PK thank the Council for Scientific and Industrial Research (CSIR), India and National Institute of Plant Genome Research (NIPGR), New Delhi, India for funding and grants. AKS thanks NIPGR for core grants.

\section{Funding}

This study was funded by Science and Engineering Research Board (SERB), Department of Science and Technology (DST), Govt. of India, for National-Post Doctoral Fellowship (NPDF) to AKS (NPDF/2015/000232).

\section{Availability of data and materials}

The details of the dataset used for the study and conclusion are included as tables (Tables 1,2 and 3) in the manuscript. The annotations of MIR394s orthologs from the newly sequenced genomes is elaborated in our manuscript and have not yet been submitted in any public databases maintained by third party. The datasets used and/or analysed during the current study are available from the corresponding author on request.

\section{Authors' contributions}

AK contributed to the retrieval, analysis and interpretation of the data, and drafting of manuscript. VG and SM contributed to analysis of data and revision of the manuscript. PK and SV contributed to revision of the manuscript. AKS contributed to conception and design of the study, interpretation of the data, enrichment and revision of manuscript. All authors read and approved the final manuscript.

\section{Ethics approval and consent to participate}

Not applicable.

\section{Consent for publication}

Not applicable.

\section{Competing interests}

The authors declare that they have no competing interest.

\section{Publisher's Note}

Springer Nature remains neutral with regard to jurisdictional claims in published maps and institutional affiliations.

\section{Received: 21 March 2018 Accepted: 5 February 2019}

\section{Published online: 14 February 2019}

\section{References}

1. Chen X. Small RNAs and their roles in plant development. Annu Rev Cell Dev Biol. 2009:25:21-44.

2. Chen $X$, Liang H, Zhang J, Zen $K$, Zhang CY. Secreted microRNAs: a new form of intercellular communication. Trends Cell Biol. 2012;22(3):125-32.

3. Jones-Rhoades MW, Bartel DP, Bartel B. MicroRNAS and their regulatory roles in plants. Annu Rev Plant Biol. 2006:57:19-53.

4. Singh A, Gautam V, Singh S, Sarkar Das S, Verma S, Mishra V, Mukherjee S, Sarkar AK. Plant small RNAs: advancement in the understanding of biogenesis and role in plant development. Planta. 2018;248(3):545-58.

5. Axtell MJ, Snyder JA, Bartel DP. Common functions for diverse small RNAs of land plants. Plant Cell. 2007;19(6):1750-69.

6. Jones-Rhoades MW, Bartel DP. Computational identification of plant microRNAs and their targets, including a stress-induced miRNA. Mol Cell. 2004;14(6):787-99.

7. Axtell MJ. Classification and comparison of small RNAs from plants. Annu Rev Plant Biol. 2013;64:137-59.

8. Chen K, Rajewsky N. The evolution of gene regulation by transcription factors and microRNAs. Nat Rev Genet. 2007;8(2):93-103.
9. Barik S, SarkarDas S, Singh A, Gautam V, Kumar P, Majee M, Sarkar AK. Phylogenetic analysis reveals conservation and diversification of micro RNA166 genes among diverse plant species. Genomics. 2014;103(1):114-21.

10. Barik S, Kumar A, Sarkar Das S, Yadav S, Gautam V, Singh A, Singh S, Sarkar AK. Coevolution pattern and functional conservation or divergence of miR167s and their targets across diverse plant species. Sci Rep. 2015;5:14611.

11. Kumar A, Sarkar AK. Apple CALCINEURIN B-LIKE PROTEIN10 genes have evolved to be novel targets of miR167s through sequence variation. Curr Sci. 2017;112.

12. Sarkar AK, Luijten M, Miyashima S, Lenhard M, Hashimoto T, Nakajima $K$, Scheres B, Heidstra R, Laux T. Conserved factors regulate signalling in Arabidopsis thaliana shoot and root stem cell organizers. Nature. 2007; 446(7137):811-4.

13. Laux T. The stem cell concept in plants: a matter of debate. Cell. 2003; 113(3):281-3.

14. Aichinger E, Kornet N, Friedrich T, Laux T. Plant stem cell niches. Annu Rev Plant Biol. 2012;63:615-36.

15. Knauer S, Holt AL, Rubio-Somoza I, Tucker EJ, Hinze A, Pisch M, Javelle M, Timmermans MC, Tucker MR, Laux T. A protodermal miR394 signal defines a region of stem cell competence in the Arabidopsis shoot meristem. Dev Cell. 2013:24(2):125-32.

16. Song JB, Huang SQ, Dalmay T, Yang ZM. Regulation of LEAF morphology by microRNA394 and its target LEAF CURLING RESPONSIVENESS. Plant and cell physiology. 2012;53(7):1283-94

17. Litholdo CG Jr, Parker BL, Eamens AL, Larsen MR, Cordwell SJ, Waterhouse PM. Proteomic identification of putative MicroRNA394 target genes in Arabidopsis thaliana identifies major latex protein family members critical for Normal development. Molecular and cellular proteomics : MCP. 2016; 15(6):2033-47.

18. Song JB, Gao S, Sun D, Li H, Shu XX, Yang ZM. miR394 and LCR are involved in Arabidopsis salt and drought stress responses in an abscisic aciddependent manner. BMC Plant Biol. 2013;13:210.

19. Song JB, Gao S, Wang Y, Li BW, Zhang YL, Yang ZM: miR394 and its target gene LCR are involved in cold stress response in Arabidopsis. Plant Gene 2016, 5(Supplement C):56-64.

20. Parry $\mathrm{G}$, Calderon-Villalobos $\mathrm{LI}$, Prigge $\mathrm{M}$, Peret B, Dharmasiri S, Itoh $\mathrm{H}$, Lechner E, Gray WM, Bennett M, Estelle M. Complex regulation of the TIR1/ AFB family of auxin receptors. Proc Natl Acad Sci. 2009;106(52):22540-5.

21. Lima JC, Arenhart RA, Margis-Pinheiro M, Margis R. Aluminum triggers broad changes in microRNA expression in rice roots. Genet Mol Res: GMR. 2011; 10(4):2817-32.

22. Gagne JM, Downes BP, Shiu SH, Durski AM, Vierstra RD. The F-box subunit of the SCF E3 complex is encoded by a diverse superfamily of genes in Arabidopsis. Proc Natl Acad Sci U S A. 2002;99(17):11519-24.

23. Skaar JR, Pagan JK, Pagano M. Mechanisms and function of substrate recruitment by F-box proteins. Nat Rev Mol Cell Biol. 2013;14(6):369-81.

24. Zheng N, Schulman BA, Song L, Miller JJ, Jeffrey PD, Wang P, Chu C, Koepp DM, Elledge SJ, Pagano M, et al. Structure of the Cul1-Rbx1-Skp1-F boxSkp2 SCF ubiquitin ligase complex. Nature. 2002:416(6882):703-9.

25. Xu G, Ma H, Nei M, Kong $\mathrm{H}$. Evolution of F-box genes in plants: different modes of sequence divergence and their relationships with functional diversification. Proc Natl Acad Sci U S A. 2009;106(3):835-40.

26. Vierstra RD. The ubiquitin-265 proteasome system at the nexus of plant biology. Nat Rev Mol Cell Biol. 2009;10(6):385-97.

27. Song JB, Shu XX, Shen Q, Li BW, Song J, Yang ZM. Altered fruit and seed development of transgenic rapeseed (Brassica napus) over-expressing MicroRNA394. PLoS One. 2015;10(5):e0125427.

28. Sunkar R, Jagadeeswaran G. In silico identification of conserved microRNAs in large number of diverse plant species. BMC Plant Biol. 2008;8:37.

29. Tian Y, Tian Y, Luo X, Zhou T, Huang Z, Liu Y, Qiu Y, Hou B, Sun D, Deng H, et al. Identification and characterization of microRNAs related to salt stress in broccoli, using high-throughput sequencing and bioinformatics analysis. BMC Plant Biol. 2014;14:226.

30. Chai J, Feng R, Shi H, Ren M, Zhang Y, Wang J. Bioinformatic identification and expression analysis of Banana MicroRNAs and their targets. PLoS One. 2015;10(4):e0123083

31. Achkar NP, Cambiagno DA, Manavella PA. miRNA biogenesis: a dynamic pathway. Trends Plant Sci. 2016;21(12):1034-44.

32. Han $Y, X u$ GX, Lu H, Yu DH, Ren Y, Wang L, Huang XH, Hou WJ, Wei ZH, Chen $Y$, et al. Dysregulation of miRNA-21 and their potential as biomarkers for the diagnosis of cervical cancer. Int J Clin Exp Pathol. 2015;8(6):7131-9. 
33. Pink RC, Samuel P, Massa D, Caley DP, Brooks SA, Carter DR. The passenger strand, miR-21-3p, plays a role in mediating cisplatin resistance in ovarian cancer cells. Gynecol Oncol. 2015;137(1):143-51.

34. Tyler DM, Okamura K, Chung WJ, Hagen JW, Berezikov E, Hannon GJ, Lai EC. Functionally distinct regulatory RNAs generated by bidirectional transcription and processing of microRNA loci. Genes Dev. 2008;22(1):26-36.

35. Schmutz J, Cannon SB, Schlueter J, Ma J, Mitros T, Nelson W, Hyten DL, Song Q, Thelen JJ, Cheng J, et al. Genome sequence of the palaeopolyploid soybean. Nature. 2010;463(7278):178-83.

36. Albert VA, Soltis DE, Carlson JE, Farmerie WG, Wall PK, llut DC, Solow TM, Mueller $L A$, Landherr $L L$, Hu $Y$, et al. Floral gene resources from basal angiosperms for comparative genomics research. BMC Plant Biol. 2005;5:5.

37. Amborella Genome P. The Amborella genome and the evolution of flowering plants. Science. 2013;342(6165):1241089.

38. Kinoshita N, Wang H, Kasahara H, Liu J, Macpherson C, Machida Y, Kamiya Y, Hannah MA, Chua NH. IAA-ala Resistant3, an evolutionarily conserved target of miR167, mediates Arabidopsis root architecture changes during high osmotic stress. Plant Cell. 2012;24(9):3590-602.

39. Lin WY, Lin YY, Chiang SF, Syu C, Hsieh LC, Chiou TJ. Evolution of microRNA827 targeting in the plant kingdom. The New phytologist. 2017.

40. Sarkar AK, Mayandi K, Gautam V, Barik S, Sarkar Das S. Improving the plant root system architecture to combat abiotic stresses incurred as a result of global climate changes. In: Climate change and plant abiotic stress tolerance: Wiley-VCH Verlag GmbH \& Co. KGaA; 2013. p. 305-24.

41. Lowe TM, Eddy SR. tRNAscan-SE: a program for improved detection of transfer RNA genes in genomic sequence. Nucleic Acids Res. 1997;25(5):955-64.

42. Lagesen K, Hallin P, Rodland EA, Staerfeldt HH, Rognes T, Ussery DW. RNAmmer: consistent and rapid annotation of ribosomal RNA genes. Nucleic Acids Res. 2007;35(9):3100-8.

43. Tempel S, Tahi F. A fast ab-initio method for predicting miRNA precursors in genomes. Nucleic Acids Res. 2012;40(11):e80

44. Tav C, Tempel S, Poligny L, Tahi F. miRNAFold: a web server for fast miRNA precursor prediction in genomes. Nucleic Acids Res. 2016;44(W1):W181-4.

45. Gruber AR, Lorenz R, Bernhart SH, Neuböck R, Hofacker IL. The Vienna RNA Websuite. Nucleic Acids Res. 2008:36(suppl_2):W70-4.

46. Zuker M, Stiegler P. Optimal computer folding of large RNA sequences using thermodynamics and auxiliary information. Nucleic Acids Res. 1981; 9(1):133-48

47. McCaskill JS. The equilibrium partition function and base pair binding probabilities for RNA secondary structure. Biopolymers. 1990;29(6-7):1105-19.

48. Nicholas HB Jr, McClain WH. Searching tRNA sequences for relatedness to aminoacyl-tRNA synthetase families. J Mol Evol. 1995;40(5):482-6.

49. Tamura K, Stecher G, Peterson D, Filipski A, Kumar S. MEGA6: molecular evolutionary genetics analysis version 6.0. Mol Biol Evol. 2013;30(12):2725-9.

50. Dai X, Zhao PX. psRNATarget: a plant small RNA target analysis server Nucleic Acids Res. 2011:39(Web Server issue):W155-9.

Ready to submit your research? Choose BMC and benefit from:

- fast, convenient online submission

- thorough peer review by experienced researchers in your field

- rapid publication on acceptance

- support for research data, including large and complex data types

- gold Open Access which fosters wider collaboration and increased citations

- maximum visibility for your research: over $100 \mathrm{M}$ website views per year

At $\mathrm{BMC}$, research is always in progress.

Learn more biomedcentral.com/submissions 